\title{
Prospects of application of mass-produced GNSS modules for solving high-precision navigation tasks
}

\author{
Vladimir Karetnikov ${ }^{1, *}$, Denis Milyakov ${ }^{2}$, Andrei Prokhorenkov ${ }^{1}$, and Evgeniy Ol'khovik ${ }^{1}$ \\ ${ }^{1}$ Admiral Makarov State University of Maritime and Inland Shipping 5/7, Dvinskaya str, Saint- \\ Petersburg, Russia, 198035 \\ ${ }^{2}$ Saint-Petersburg branch of Navis Inc, Russia
}

\begin{abstract}
Nowadays, both in the Russian Federation and in foreign countries, the use of global navigation satellite systems (GNSS) for solving various applied problems is an extremely popular solution. Taking into account the current level of development of satellite radio navigation systems, ordinary users have been able to determine their position with a sufficiently high accuracy. However, some tasks require the use of highprecision equipment of geodetic class. Such equipment allows obtaining navigation solutions with an accuracy of better than $10 \mathrm{~cm}$. Unfortunately, the cost of navigation-class navigation equipment is extremely high. Specialists working in the field of satellite navigation are particularly interested in the possibility of using mass-produced GNSS modules to obtain high-precision navigation solutions. In this paper, the possibility of such an application will be considered, taking into account the results of laboratory tests of a mass-produced navigation module.
\end{abstract}

\section{Introduction}

In accordance with the Federal Target Program "Maintenance, Development and Use of the GLONASS Global Navigation Satellite System for 2012-2020", one of the most important tasks was the development of complementary GLONASS GNSS complexes, including the creation of systems for high-precision determination of navigation and ephemeris-time information. The area for the development of high-precision navigation systems based on GLONASS GNSS signals receives support not only in the field of public administration, but also in the commercial sector of the economy, which, given the negative foreign policy environment and sanctions, is an incentive for the creation of high-tech highprecision navigation equipment [1].

Despite the fact that the development of the domestic market for the electronic industry and, as a consequence, high-tech navigation equipment lags behind Western companies, and many experts in the field of satellite navigation believe that it is not worth setting the goal of developing the GLONASS GNSS system to catch up with the developers of GNSS

\footnotetext{
*Corresponding author: karetnikovvv@gumrf.ru
} 
GPS in accuracy, since the current accuracy is enough for most tasks, the development of the fleet of high-precision navigation equipment in the Russian Federation continues [2].

Determination of coordinates with a centimeter level of accuracy still requires the use of expensive GNSS consumer navigation equipment (CNE) [3]. However, at present, the basic elements of high-precision positioning technology using mass-produced GNSS modules have been developed and tested, which in the future, most likely, will make high-precision positioning available to almost any owner of a smartphone or, for example, an auto navigator. This will inevitably radically change the markets for geodesic and navigation equipment and geoinformation.

\section{Materials and Methods}

In 2019, KB NAVIS JSC developed a prototype of a set of high-precision navigation equipment (HPNE), operating according to GLONASS/GPS/GALILEO/BEIDOU GNSS signals, with the function of receiving assistive data via a radio channel. HPNE equipment performs high-precision positioning in absolute mode (PPP mode - Precise Point Positioning) [4,5] by joint processing of two-frequency measurements (pseudo-ranges and pseudophases) and high-precision ephemeris-time information (ETI) [6] of real-time GNSS in the form of RTCM 10403.2 standard coming from the Ministry of Defense Precision Navigation System (MD PNS).

HPNE provides continuous tracking of GNSS GLONASS navigation spacecraft and conduct code and phase measurements by signals [7]:

1. GLONASS:

- high precision with frequency division: L1SF, L2SF;

- high precision code division: L1SC, L2SC;

- standard precision with frequency division: L1OF, L2OF;

- standard precision code division: L1OC, L2OC, L3OC.

2. GPS - L1 C/A, L1C, L2C, L5C;

3. Galileo - E1bc, E5a, E5 в;

4. Beidou - B1I, B2I.

The HPNE set includes:

- navigation box with built-in rechargeable battery;

- antenna unit;

- charger;

- a set of cables and accessories.

Software developed to implement PPP mode in the prototype of HPNE provides:

- determination of absolute coordinates, components of the velocity vector of the ground observer and corrections to the receiver time scale in real time (navigation mode);

- processing of dual-frequency phase and code measurements of GNSS (IonoFree combinations) in real time using high-precision assistance information coming from the MD PNS (high-precision PPP determination mode);

- registration of primary code and phase measurements with the purpose of subsequent transmission to the Center for Precision Information (CPI-M) of MD PNS (base station mode);

- taking into account and correcting the main factors of observation errors, taking into account the recommendations of the IGS and the system for high-precision determination of ephemerid-time corrections (SHPD ETC).

High-precision determination of coordinates in real time is carried out using modern algorithms for joint processing of GNSS signals and assistance information. 


\section{Results}

Solving coordinate-time tasks in PPP mode using assistance information.

The computational resources of HPNE (Dolomant's computing module operating at 600 $\mathrm{MHz}$ ) make it possible to use recurrent navigation filters that estimate coordinates, velocities and phase ambiguities, as well as tropospheric parameters (the wet component of the vertical propagation delay of the navigation signal). The traditional approach to the precision solution of the navigation problem for mobile equipment is the use of extended Kalman filters (EKF) [8] and their various modifications.

As part of the work performed, a software multisystem PPP navigation algorithm based on the Kalman filter was developed and implemented, which can be successfully used to solve both static and dynamic problems of coordinate determination.

As an observation vector in such a filter, measurements of "ionospheric-free" combinations of pseudo-ranges and phases are used [9]; the estimated parameters include corrections to the coordinates, the receiver time scale, the values of phase ambiguities for the used phase combinations, as well as the total vertical tropospheric delay or its wet part.

The inclusion of phase ambiguities in the estimated parameters makes it possible to use the entire volume of available phase measurement information in their estimation, and not only distance measurements of the corresponding channel, which makes it possible to increase both the accuracy of the solution and the rate of its convergence. The downside is the significant increase in computational costs caused by the increase in dimension.

The traditional algorithm includes two cyclically repeated main steps, commonly called prediction and correction. Below, for an example, an algorithm for a single-system implementation is given, corresponding to autonomous operation on the GLONASS GNSS system:

Stage "Prediction"

$$
\begin{gathered}
X_{i+1}^{\prime}=\Phi X_{i} . \\
P_{i+1}^{\prime}=\Phi P_{i} \Phi^{T}+Q .
\end{gathered}
$$

Stage "Correction”

$$
\begin{gathered}
K_{i+1}=\frac{P_{i+1} H_{i+1}^{T}}{H_{i+1} P_{i+1} H_{i+1}^{T}+W} . \\
P_{i+1}=P_{i+1}^{\prime}-K_{i+1} H_{i+1} P_{i+1}^{\prime} . \\
X_{i+1}=X_{i+1}^{\prime}+K_{i+1}\left(R_{i+1}-R\left(X_{i+1}^{\prime}\right)\right) .
\end{gathered}
$$

Here the vector of observations:

$$
R=\operatorname{col}\left(D_{1} \ldots D_{N}, L_{1} \ldots L_{N}\right),
$$

where $D_{i}-$ measurements of "ionosphere-free" combinations of pseudo-ranges and $L_{i}-$ similar combinations of total phases.

Vector of estimated parameters:

$$
X=\operatorname{col}\left(D_{X}, D_{Y}, D_{Z}, D_{T}, V_{T}, N_{1} \ldots N_{N}\right),
$$


Where $\mathrm{D}_{\mathrm{X}}, \mathrm{D}_{\mathrm{Y}}, \mathrm{D}_{\mathrm{Z}}, \mathrm{D}_{\mathrm{T}}$ - corrections to navigation coordinates and receiver scale, $\mathrm{V}_{\mathrm{T}}-$ vertical tropospheric delay, or its wet part, $\mathrm{N}_{1} \ldots \mathrm{N}_{\mathrm{N}}$ - values of phase multivalues for $\mathrm{N}$ used satellites.

Matrix $\Phi$ defines the model of dynamics with model noise Q, P - the covariance matrix of the estimate of the desired vector $\mathrm{X}$, the observation noise is characterized by the matrix $\mathrm{W}$.

$\mathrm{H}$ - gradient matrix:

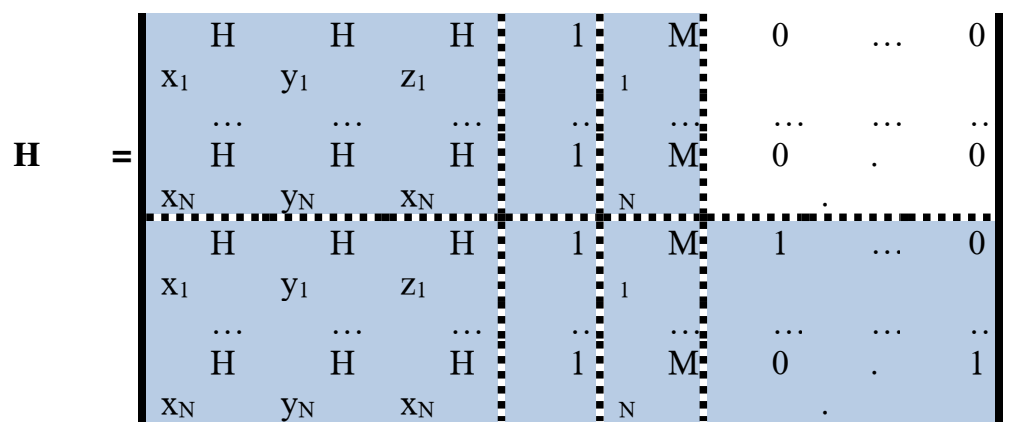

Here $\mathrm{H}_{\mathrm{x}}, \mathrm{H}_{\mathrm{y}}, \mathrm{H}_{\mathrm{z}}$ - direction cosines on satellites, $\mathrm{M}_{\mathrm{i}}$ - values of the tropospheric display function for the corresponding satellite.

In the case of an a priori unknown (stochastic) law of motion, as corrections to the coordinates, it is advisable to seek corrections to the previously obtained conventional navigation solution based on single observations. In this case, matrix Q will characterize the coordinate noise of this navigation solution. Then the identity matrix can be used as matrix $\Phi$, except for the diagonal element corresponding to the estimate of the wet delay. I.e.:

$$
\Phi=\operatorname{diag}\left(1,1,1,1, \exp \left(\frac{-T_{\text {emp }}}{T_{\text {corr }}}\right), 1 \ldots 1\right)
$$

where Темр - rate of data receipt and processing, Tcorr - the time constant of the correlation of the errors of the "wet" part of the vertical tropospheric delay (usually selected in the range of 1-3 hours).

In this case, the result of solving the traditional navigation problem is used as the extrapolated $\mathrm{D}_{\mathrm{X}}, \mathrm{D}_{\mathrm{Y}}, \mathrm{D}_{\mathrm{Z}}, \mathrm{D}_{\mathrm{T}}$. This scheme makes the EKF algorithm completely independent of the consumer dynamics and the used model for predicting the coordinate components of the vector of the estimated parameters. At the same time, such a scheme is not optimal for many applications, since the use of more complex dynamic models for extrapolation (with a corresponding expansion of the vector of estimated parameters due to the corresponding derivatives of coordinates) or additional information from inertial sensors will further narrow the filter tracking zone and increase the accuracy of the assessment.

\section{Discussion}

To optimize the solution of the coordinate problem and accelerate the convergence, it is important to correctly select the elements of the observation weights matrix W. It must be recalculated dynamically and can take into account the change in measurement accuracy depending on the signal-to-noise ratio, elevation angle, pre-smoothing time for distance measurements (if used), and other factors. The traditional, simplest and most frequently implemented approach involves the use of the value of functions of the elevation angle as 
weighting coefficients. Correct formation of the weight matrix increases the rate of convergence of the solution and increases its accuracy, especially in dynamic applications. The following rule is usually used to form the weight matrix:

$$
\begin{gathered}
W=\operatorname{diag}\left(W_{1} \ldots W_{N}\right), W_{i}(a)=C_{0}^{2}, a>a_{0} . \\
W_{i}(a)=\frac{C_{0}^{2} \sin ^{2}(a)}{\sin ^{2}(a)}, a \leq a_{0} .
\end{gathered}
$$

Here a-elevation angle of the observed i-th satellite. $\mathrm{C}_{0}{ }^{2}$ - nominal measurement noise, angle $\mathrm{a}_{0}$ is chosen, as a rule, empirically.

The hardware implements two modes of PPP definitions - static $[10,11]$ and dynamic. The main differences between static (used for static initialization) and dynamic algorithms are as follows:

1. In the static algorithm, the corrections to the constant initial approximation of the station coordinates are estimated, in the dynamic algorithm - to the results of the navigation solution obtained from the measurements of pseudo-ranges.

2. In the static algorithm, the influence of lithospheric dynamics is removed from the corrections (null-tide solution), in the dynamic one, this influence is kept.

3. Differences in the thresholds for rejecting faulty measurements (for statics, rejection is more severe).

When processing the data, accounting and correction of the main error factors was ensured, taking into account the recommendations of IGS and SHPD ETC:

- two-frequency combinations of phase measurements were used, which ensured the elimination of the ionospheric error;

- high-precision ephemeris-time information of real time in the format of the RTCM 10403.2 (SSR) standard, coming from the Ministry of Defense precision navigation system (MD PNS) and/or other similar sources, was used;

- the relativistic SRT and GRT corrections and the Wind-Up effect were taken into account;

- to correct the hydrostatic part of the tropospheric delay, the Saastamoinen-Davis troposphere model was used, taking into account the course of temperatures, humidity and pressure according to the ICAO seasonal atmospheric model;

- when estimating the wet part of the tropospheric delay, the GMF display function was used;

- for static problems, tidal phenomena in the lithosphere, caused by the influence of the Sun and the Moon, as well as by the movements of the Earth's pole, were taken into account and compensated for;

- additional control of undiagnosed disruptions of phase measurements (the main method was control of the smoothness of the phase differences L1 and L2) and rejection of gross errors (RAIM) were made.

Below are some of the HPNE test results, including those obtained on the models of the equipment.

The values of the error (with a probability of 0.68 ) for determining coordinates in real time in PPP mode and PPP mode with initialization when using the data of the assistance information of the SHPD ETC or similar sources of high-precision ETI are given in Table 1 and Table 2. 
Table 1. Values of the error (with a probability of 0.68 ) for determining the coordinates in real time in the PPP mode when using the assistance information data (after the completion of the transient process).

\begin{tabular}{|l|c|c|c|}
\hline \multirow{2}{*}{$\begin{array}{c}\text { GNSS signal } \\
\text { reception mode }\end{array}$} & \multicolumn{3}{|c|}{ Error (with a probability of $\mathbf{0 . 6 8}$ ) in determining coordinates, $\mathbf{m}$} \\
\cline { 2 - 4 } & $\mathbf{x}$ & $\mathbf{y}$ & $\mathbf{H}$ \\
\hline GLONASS GPS, & 0.14 & 0.12 & 0.26 \\
\hline $\begin{array}{l}\text { GLONASS, BEIDOU } \\
\text { GALILEO, BEI }\end{array}$ & 0.12 & 0.12 & 0.16 \\
\hline
\end{tabular}

Table 2. Error values (with a probability of 0.68 ) for determining coordinates in real time in PPP mode with initialization when using the assistance information data (after completion of static initialization).

\begin{tabular}{|l|c|c|c|}
\hline \multirow{2}{*}{$\begin{array}{c}\text { GNSS signal } \\
\text { reception mode }\end{array}$} & \multicolumn{3}{|c|}{ Error (with a probability of $\mathbf{0 . 6 8}$ ) in determining coordinates, $\mathbf{m}$} \\
\cline { 2 - 4 } & $\mathbf{x}$ & $\mathbf{y}$ & $\mathbf{H}$ \\
\hline GLONASS GPS, & 0.07 & 0.06 & 0.18 \\
\hline $\begin{array}{l}\text { GLONASS, BEIDOU } \\
\text { GALILEO, BEI }\end{array}$ & 0.06 & 0.06 & 0.10 \\
\hline
\end{tabular}

Below is shown the typical behavior of the HPNE coordinate solution during operation and the results of processing absolute coordinate determinations in real time.

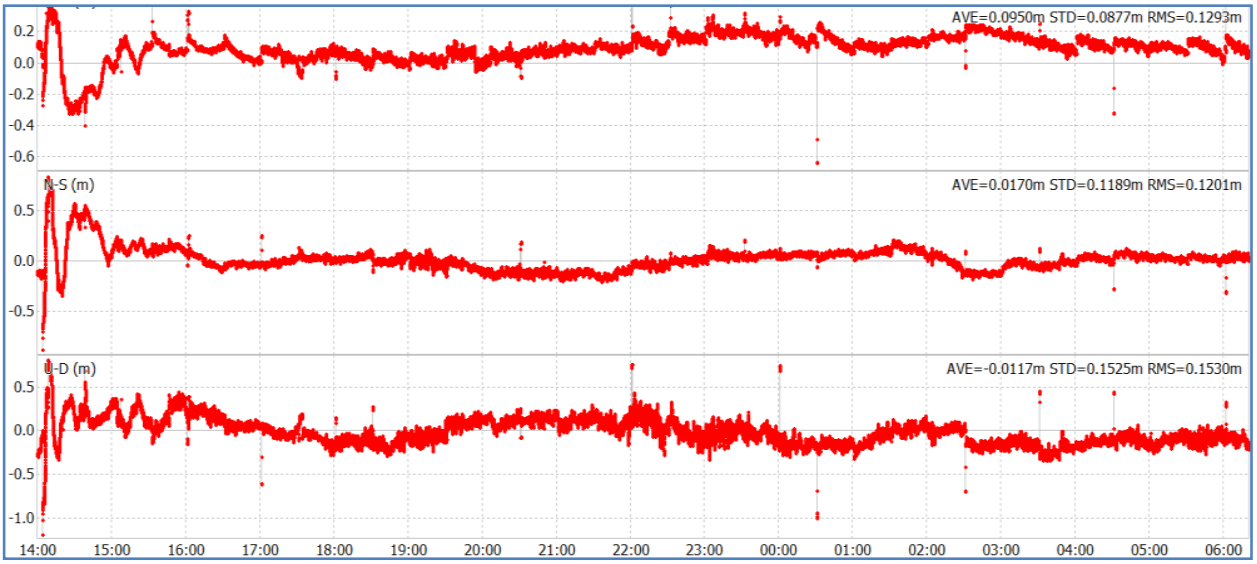

Fig. 1. Values of errors of positioning by GNSS GLONASS + GPS in PPP mode in real time.

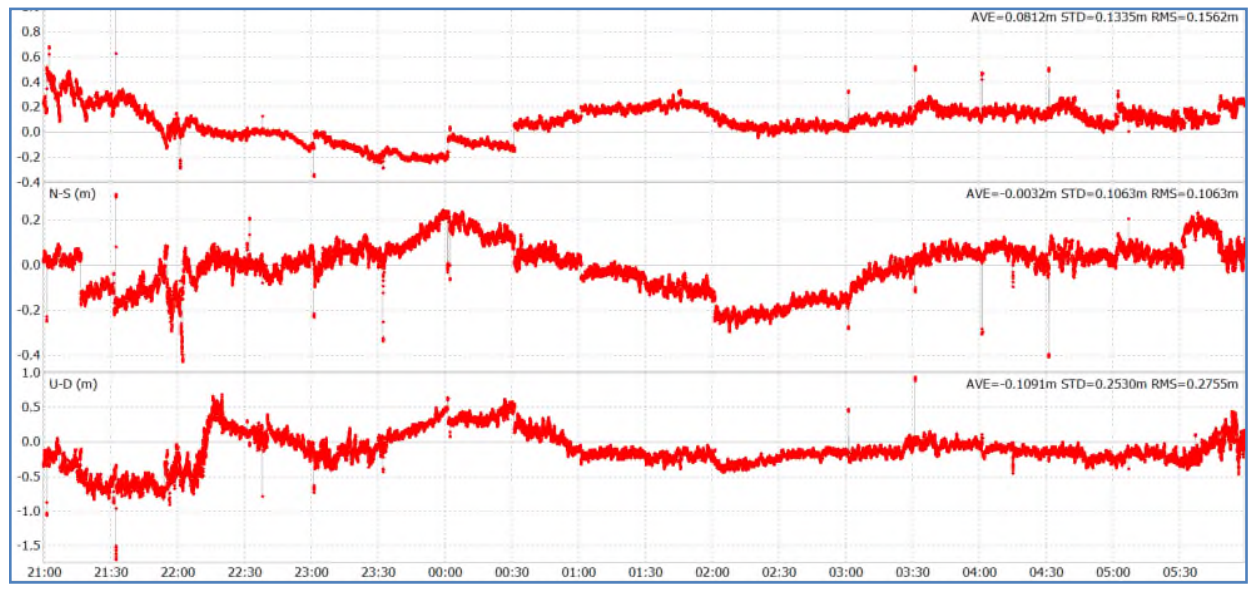

Fig. 2. Values of errors of definitions by GNSS GLONASS in PPP mode in real time. 
Conclusions based on the results of testing HPNE.

1. The Kalman filtering algorithms implemented in HPNE provide the solution of static and dynamic problems of PPP-definitions, including those using measurements only by the GLONASS system.

2. When working in real time, including only by GNSS GLONASS, the error values are achieved at the level of $0.1-0.15 \mathrm{~m}$ in plain and $0.2-0.3 \mathrm{~m}$ in height.

3 . When carrying out static initialization, the time of convergence of the navigation solution in dynamics to a level of $10 \mathrm{~cm}$ with a discreteness of $1 \mathrm{sec}$ is $0.6-1.0$ hours.

\section{Conclusion}

The social effect of the introduction of high-precision positioning technologies with the help of massively available consumer equipment is obvious in increasing labor productivity in various fields of activity associated with the creation and use of geo-information.

Low-budget consumer equipment providing such a level of accuracy can be widely used in solving various practical problems, namely:

- solving many problems of engineering geodesy;

- ensuring high-precision control of traffic and the development of unmanned vehicles;

- the use of automated control systems for equipment;

- control of dynamic deformations of technical structures;

- precise personal navigation (can be used, for example, when searching for small inconspicuous or hidden under the ground, water or snow objects: underground or underwater communications, wells, etc. - at industrial sites, railway stations, energy facilities, utilities, etc.); and others.

\section{References}

1. V. Karetnikov, S. Rudykh, A. Ivanova, MATEC Web of Conferences 265, 02016 (2019)

2. V. Karetnikov, I. Pashchenko, V. Kozlov, A. Butsanets, IOP Conference Series: Materials Science and Engineering 811, 012005 (2020)

3. V. Karetnikov, E. Ol'Khovik, A. Ivanova, A. Butsanets, Intelligent Systems and Computing 1258 AISC, 421-432 (2021)

4. S. Savchuk, J. Cwiklak, A. Khoptar, Baltic Surveying 12, 39-43 (2020)

5. S. Bulbul, B. Bilgen, C. Inal, Measurement 171, 108780 (2021)

6. I.V. Bezmenov, Measurement Techniques 63(1), 7-14 (2020)

7. A. Karutin, Proceedings of the 33rd International Technical Meeting of the Satellite Division of The Institute of Navigation (ION GNSS+ 2020) (2020)

8. A. Elmezayen, A. El-Rabbany, Survey Review, 1-15 (2020)

9. F. Basile, T. Moore, C. Hill, G. McGraw, Journal of Navigation 74(1), 5-23 (2020)

10. O. Sterle, B. Stopar, P. Pavlovčič Prešeren, Geodetski vestnik 58(03), 466-481 (2014)

11. M.A. Rabbou, A. El-Rabbany, Positioning 06(01), 1-6 (2015) 